\title{
Diagnostic performance of APRI and FIB-4 for confirming cirrhosis in Indonesian HIV/ HCV co-infected patients
}

Evy Yunihastuti ${ }^{1,2^{*}}$ (D, Bramantya Wicaksana ${ }^{1}$, Andrian Wiraguna', Ainum Jhariah Hidayah' ${ }^{1}$, Fhadilla Amelia', Veritea Natali ${ }^{1}$, Alvina Widhani ${ }^{1,2}$, Andri Sanityoso Sulaiman ${ }^{2}$ and Juferdy Kurniawann ${ }^{2}$

\begin{abstract}
Background: After successful of antiretroviral therapy, highly effective direct acting antiviral (DAA) make HCV elimination reasonable in HIV/HCV co-infected patients. However, in achieving this target, there are still barriers to start DAA treatment, particularly in the area of liver fibrosis assessment that determine the duration of therapy. We aimed to assess the diagnostic performance of APRI and FIB-4 for diagnosing cirrhosis in HIV/HCV co-infected patients using hepatic transient elastography (TE) as gold standard.

Method: This is a retrospective study on HIV/HCV co-infected patients who concomitantly performed hepatic TE measurement, APRI, and FIB-4 evaluation before HCV treatment initiation at a tertiary hospital in Jakarta from 2014 to 2019. Sensitivity, specificity and diagnostic accuracy of indirect biomarkers for liver stiffness measurement $(\mathrm{LSM}) \geq 12.5 \mathrm{kPa}$ was determined by receiver operator characteristics curves.

Results: $223 \mathrm{HIV} / \mathrm{HCV}$ co-infected patients on stable antiretroviral therapy were included, of whom 91.5\% were male with mean age of 37 (SD 5) years. Only $28.7 \%$ of patients were classified as cirrhosis (F4). Using TE as gold standard ( $\geq 12.5 \mathrm{kPa}$ ), the low threshold of APRI (1) had specificity $95 \%$, sensitivity $48.4 \%$, correctly classified $81.6 \%$ of patients, with moderate performance, AUC at 0.72 (95\% Cl 0.63-0.80). The optimal cut-off of FIB-4 was 1.66 [specificity $92.5 \%$, sensitivity $53.1 \%, \mathrm{AUC}$ at $0.73(95 \% \mathrm{Cl} 0.65-0.81)$ ] and correctly classified $81.1 \%$ of the patients.

Conclusion: APRI score $\geq 1$ and FIB-4 score $\geq 1.66$ had moderate performance with high specificity in diagnosing cirrhosis. These biochemical markers could be used while TE is not available.
\end{abstract}

Keywords: APRI, FIB-4, Transient elastography, HIV, HCV

\section{Background}

Hepatitis C virus (HCV infection) is one of global health problem that can increase morbidity and mortality in HIV-infected population. In 2017, about 71 million people living with chronic $\mathrm{HCV}$ infection globally, of which only $19 \%$ (13.1 million) knew their diagnosis. The estimated prevalence in South-East Asia was $0.5 \%$ with

\footnotetext{
* Correspondence: evy.yunihastuti@gmail.com

${ }^{1}$ HIV integrated services, Cipto Mangunkusumo Hospital, Jakarta, Indonesia ${ }^{2}$ Department of Internal Medicine, Faculty of Medicine Universitas Indonesia, Cipto Mangunkusumo Hospital, Diponegoro, Jakarta 71, Indonesia
}

incidence 14.8 (95\% CI 12.5-26.9) per 100,000 population in 2015 [1]. In Indonesia, HCV infection prevalence is estimated at $1 \%$, of which $90 \%$ comes from intravenous drug user [2]. World Health Organization (WHO) recently released targets for $\mathrm{HCV}$ elimination by 2030 , targeting 80\% HCV-infected patients being treated [1]. A large gap between $\mathrm{HCV}$-diagnosed patients and $\mathrm{HCV}$ treated patients still exist to achieve this global target since only around $10 \%$ of $\mathrm{HCV}$-infected patients in South East Asia have been treated [3]. Achieving global elimination of $\mathrm{HCV}$ need major increase in service all

(c) The Author(s). 2020 Open Access This article is licensed under a Creative Commons Attribution 4.0 International License, which permits use, sharing, adaptation, distribution and reproduction in any medium or format, as long as you give appropriate credit to the original author(s) and the source, provide a link to the Creative Commons licence, and indicate if changes were made. The images or other third party material in this article are included in the article's Creative Commons licence, unless indicated otherwise in a credit line to the material. If material is not included in the article's Creative Commons licence and your intended use is not permitted by statutory regulation or exceeds the permitted use, you will need to obtain permission directly from the copyright holder. To view a copy of this licence, visit http://creativecommons.org/licenses/by/4.0/ The Creative Commons Public Domain Dedication waiver (http://creativecommons.org/publicdomain/zero/1.0/) applies to the data made available in this article, unless otherwise stated in a credit line to the data. 
populations along the entire cascade of care, including testing, linkage to care, retention in care, treatment, chronic care and prevention of primary infection and reinfection [4]. In 2017, Indonesian government started free direct acting antiviral (DAA) program for $\mathrm{HCV}$, mainly sofosbuvir and daclatasvir combination [2, 5]. This 12 weeks combination for non-cirrhotic patients and 24 weeks for cirrhotic-patients shown similar success rate for both $\mathrm{HIV} / \mathrm{HCV}$ co-infected patients and HCV mono-infected patients [6]. Despite these facts, there are still many barriers to start HCV treatment, especially in defining liver fibrosis phase. Liver biopsy has been gold standard for liver fibrosis assessment for a long time. This invasive procedure carries some serious side effects and can only be done in specialized care. Transient elastography (TE) is a non-invasive method that measures liver stiffness using both ultrasound and low-frequency elastic waves to define liver fibrosis phase [7]. This method has been validated in viral hepatitis with equal performance in $\mathrm{HCV}$ and $\mathrm{HIV} / \mathrm{HCV}$ coinfection [8]. However, it is not widely available in Indonesia and still carries a high-cost examination.

Several laboratory tests, scores, and indices have been developed for non-invasive prediction of cirrhosis (F4) in HCV mono-infected patients. Aspartate aminotransferase (AST)-to- platelet ratio (APRI), fibrosis-4 (FIB4), modified APRI, Forns, and Göteborg University Cirrhosis Index (GUCI) are based on routine laboratory parameters and readily available in clinical practice. The two most common and validated used are APRI and FIB-4. WHO ranked these two scores as preferred non-invasive markers of fibrosis in resource-limited setting [9].

Since the determination of liver cirrhosis significantly influences the decision for treatment duration of sofosbuvir and daclatasvir, optimal cut-off of APRI and FIB-4 to diagnose cirrhosis need to be defined in our $\mathrm{HIV} / \mathrm{HCV}$ co-infected population. We aimed to assess the diagnostic performance of APRI and FIB-4 for diagnosing liver cirrhosis in Indonesian HIV/HCV co-infected patients using hepatic TE (Fibroscan ${ }^{\circ}$ ) as a gold standard.

\section{Methods}

\section{Study design}

A retrospective study on $\mathrm{HIV} / \mathrm{HCV}$ co-infected patients who concomitantly performed hepatic TE measurement, APRI, and FIB-4 evaluation before HCV treatment initiation at HIV Integrated Clinic, Cipto Mangunkusumo Hospital, Jakarta between 2014 and December 2019. The demographic data, clinical, and laboratory data were collected from hospital medical record. Clinical data collected including body mass index (BMI), HBsAg seropositivity, and ART history. Laboratory data including $\mathrm{HCV}$ RNA, complete blood count (CDC), transaminase value, and estimated glomerular filtration rate (eGFR).

\section{Eligibility criteria}

Inclusion criteria were infection with $\mathrm{HCV}$ defined as a positive HCV-RNA, HIV infection on stable antiretroviral treatment, and no prior HCV treatment. Exclusion criteria was hepatocellular carcinoma.

\section{Instrumental assessment}

Liver fibrosis or cirrhosis was assessed using transient elastography (FibroScan $502^{\circ}$, Echosens, France) by trained operators according to the manufacturer's protocol. The procedures of liver stiffness measurement (LSM) were patient in a supine position, then M probe was put in intercostal spaces on the right lobe. The value of LSM was expressed in kilopascal $(\mathrm{kPa})$. Patients were recommended to do fasting before the examination. LSM values were included in this analysis with at least 10 valid measures, over $60 \%$ success rate and interquartile range (IQR) less than $30 \%$ of the median value of LSM [10]. The following cut-offs were used to stage the liver fibrosis are F0-F1 $<7.1 \mathrm{kPa}, \mathrm{F} 2$ (significant fibrosis) $7.1-9.4 \mathrm{kPa}, \mathrm{F} 3$ (advanced fibrosis) $9.5-12.4 \mathrm{kPa}$, and F4 (cirrhosis) $\geq 12.5 \mathrm{kPa}$ [11].

The APRI score was calculated using the formula: [(AST level/ULN)/platelet count $\left.\left(10^{9} / \mathrm{L}\right)\right] \times 100$ [9]. The reference upper normal limit (ULN) for AST used in our hospital was $44 \mathrm{IU}$. While the FIB-4 score was using formula: age $\mathrm{x} \mathrm{AST/(platelet} \mathrm{count} \mathrm{x} \sqrt{ } \mathrm{ALT}$ ) [9].

\section{Statistical analysis}

All statistical analysis were performed using STATA statistical package for Windows version 12.0 (Statacorp, USA). The description of socio-demographics, HIV characteristics, and $\mathrm{HCV}$ characteristics was performed using standard descriptive methods. Categorical variables were expressed as raw numbers and percentages and compared using the chi-square test, and continuous variables were described by means and standard deviation (normal distribution) or median and IQR 25-75\% (non-normal distribution assessed by Kolmogorov-Smirnov test). The proportion of liver cirrhosis $(\mathrm{F} \geq 4)$ was assessed as the number of patients with LSM of minimum $12.5 \mathrm{kPa}$ and expressed with its $95 \%$ confidence interval. A two-sided $p$ value $<0.05$ was considered to be statistically significant.

Performances of APRI and FIB-4 in diagnosing cirrhosis were evaluated from Area Under Receiver-Operator Curves (AUROCs) using binomial method, considering $\mathrm{TE}$ as the gold standard. Using WHO hepatitis $\mathrm{C}$ guidelines, two thresholds for fibrosis $(\mathrm{F} \geq 4)$ with APRI examined: 2 (high threshold) and 1 (low threshold) [9]. While the diagnosis of cirrhosis $(\mathrm{F} \geq 4)$ with FIB- 4 was examined as 1.94 and 3.25 cut-offs based on previous study in HIV/HCV co-infected patients [12]. To evaluate the diagnostic accuracy of available cut-offs of APRI and 
FIB-4, sensitivity, specificity, positive predictive value (PPV), negative predictive value (NPV), positive likelihood ratio $(\mathrm{LR}+)$, negative likelihood ratio (LR-) were estimated for each cut-off value. Accuracy was assessed by the percentage of patients that were correctly classified. In finding the novel diagnostic cut-offs for APRI and FIB-4 in our population, the ROCs were generated by plotting the relationship of the true positivity (sensitivity) and the false positivity (1-specificity) at various cutoff points of each score. We choose optimal cut-off point using to the highest rate of correctly classified patients.

\section{Results}

\section{Patient characteristics}

Of 223 study patients, $91.5 \%$ were male with a mean age of 37 (SD 5) years. All patients were under stable antiretroviral therapy (ART) medication, mostly efavirenz
(EFV)-based regiment (43\%) or nevirapine (NVP)-based regiment (42.2\%). Median absolute CD4 cell count was 508 (IQR 306) cells $/ \mathrm{mm}^{3}$ and the median duration of ART was 6.8 (IQR 4) years. Most of patients (93.3\%) had history of intravenous drug use (IVDU) and $71.3 \%$ patients reported to have past alcohol consumption. Only 8 patients $(3.6 \%)$ had all hepatitis B, hepatitis $\mathrm{C}$, and HIV infections. All demographics and HIV characteristics were similar between cirrhotic and non-cirrhotic patients, except ART-regimen used (Table 1). We did not find any decompensated cirrhosis among 64 cirrhotic patients within recruitment phase (the data was not shown).

\section{Hepatitis $\mathrm{C}$ and liver fibrosis evaluation}

In this study, mean HCV RNA viral load was 6.12 (SD 0.81) $\log _{10} \mathrm{IU} / \mathrm{mL}$, as seen in Table 1. Median LSM for all the patients was 8.8 (IQR 7.3) kPa. Cirrhotic patients

Table 1 Description of the study population according to the liver cirrhosis status

\begin{tabular}{|c|c|c|c|c|}
\hline & Total $(n=223)$ & $\begin{array}{l}\text { Non-cirrhosis } \\
(\mathrm{LSM}<12.5 \mathrm{kPa}) \\
(n=159)\end{array}$ & $\begin{array}{l}\text { Cirrhosis } \\
(\mathrm{LSM} \geq 12.5 \mathrm{kPa}) \\
(n=64)\end{array}$ & $p$ value \\
\hline \multicolumn{5}{|l|}{ Socio-demographic characteristics } \\
\hline Age, years, mean (SD) & $37(5)$ & $37(5)$ & $38(6)$ & 0.376 \\
\hline Male, n(\%) & $204(91.5)$ & $142(89.3)$ & $62(96.8)$ & 0.068 \\
\hline $\mathrm{BMI}, \mathrm{kg} / \mathrm{m}^{2}$, mean (SD) & $22.55(3.80)$ & $22.26(3.56)$ & $23.27(4.27)$ & 0.071 \\
\hline IVDU history, n(\%) & $208(93.3)$ & $148(93.1)$ & $60(93.7)$ & 0.857 \\
\hline Alcohol history, n(\%) & $159(71.3)$ & $114(71.7)$ & $45(70.3)$ & 0.836 \\
\hline HBsAg seropositivity, n(\%) & $8(3.6)$ & $7(4.4)$ & $1(1.5)$ & 0.303 \\
\hline \multicolumn{5}{|l|}{ HIV characteristics } \\
\hline ART duration, years, median (IQR) & $6.8(4)$ & $6.8(5)$ & $6.8(2)$ & 0.264 \\
\hline Current CD4, cells $/ \mathrm{mm}^{3}$, median (IQR) & $508(306)$ & $508(333)$ & $508(215)$ & 0.855 \\
\hline \multicolumn{5}{|l|}{ ART used } \\
\hline $2 \mathrm{NRTI}+\mathrm{EFV}, \mathrm{n}(\%)$ & $96(43)$ & $76(47.7)$ & $20(31.2)$ & 0.011 \\
\hline $2 \mathrm{NRTI}+\mathrm{NVP}, \mathrm{n}(\%)$ & $94(42.2)$ & $64(40.2)$ & $30(46.8)$ & \\
\hline $2 \mathrm{NRTI}+\mathrm{LPV} / \mathrm{r}, \mathrm{n}(\%)$ & $33(14.8)$ & 19 (11.9) & $14(21.8)$ & \\
\hline \multicolumn{5}{|l|}{ HCV infection characteristics } \\
\hline HCV-RNA, $\log _{10}$, mean (SD) & $6.12(0.81)$ & $6.15(0.84)$ & $6.06(0.73)$ & 0.453 \\
\hline Hemoglobin, g/dL, mean (SD) & $13.8(2.3)$ & $13.9(2.0)$ & $13.5(2.8)$ & 0.201 \\
\hline Platelet, $10^{3} / \mathrm{mL}$, median (IQR) & $225(77)$ & $249(78)$ & $173(72)$ & $<0.001$ \\
\hline ALT, U/L, median (IQR) & $55(51)$ & $50(41)$ & $66.5(71)$ & $<0.001$ \\
\hline AST, U/L, median (IQR) & $41(41)$ & $37(25)$ & $71(64)$ & $<0.001$ \\
\hline eGFR (CKD-EPI), mL/min/1.73 m², mean (SD) & $108.5(21.3)$ & $108.5(20.7)$ & $108.5(23.0)$ & 0.998 \\
\hline LSM (kPa), median (IQR) & $8.8(7.3)$ & $7.8(3.8)$ & $20.1(10.4)$ & $<0.001$ \\
\hline APRI score, median (IQR) & $0.41(0.52)$ & $0.34(0.33)$ & $0.97(1.25)$ & $<0.001$ \\
\hline FIB-4 score, median (IQR) & $0.88(0.73)$ & $0.81(0.45)$ & $1.69(1.76)$ & $<0.001$ \\
\hline
\end{tabular}

SD: standard deviation; BMI: body mass index; IVDU: intravenous drug user; NRTI: nucleoside reverse transcriptase inhibitor; EFV: efavirenz; NVP: nevirapine; LPV/r: lopinavir/ritonavir; HCV-RNA: Hepatitis C virus-ribonucleic acid; ALT: alanine aminotransferase; AST: aspartate aminotransferase; eGFR: estimated glomerular filtration rate; CKD-EPI: chronic kidney disease-epidemiology collaboration; LSM: liver stiffness measurement; APRI: AST to platelet ratio index; FIB-4: the fibrosis-4 index; IQR: interquartile range; SD: standard deviation; kPa: kilo pascal 
(28.7\% of total participants) had median LSM 20.1 (IQR 10.4) $\mathrm{kPa}$, while in non-cirrhotic patients the median LSM was 7.8 (IQR 3.8) kPa. Patients with cirrhosis had lower platelet count, higher ALT and AST level compared to non-cirrhotic patients. There was no difference between cirrhotic and non-cirrhotic patients in term of their HCV RNA viral load, hemoglobin, and kidney function (eGFR). The median values of fibrosis indices were: 0.41 (IQR 0.52) for APRI and 0.88 (IQR 0.73) for FIB-4. Higher values of APRI and FIB-4 were observed in patients with $\mathrm{LSM} \geq 12.5 \mathrm{KPa}$ compared to patients with $\mathrm{LSM}<12.5 \mathrm{KPa}$ : APRI 0.97 (IQR 1.25) vs 0.34 (IQR $0.33), p<0.001$ and FIB-4 1.69 (IQR 1.76) vs 0.81 (0.45), $p<0.001$.

Performance of APRI scores for the diagnosis of cirrhosis We evaluated two different APRI threshold as recommended by WHO. In our population, an APRI value of 2 (high threshold) shown sensitivity $18.7 \%$, specificity $98.1 \%$, positive predictive value $80 \%$, negative predictive value $75 \%$, positive likelihood ratio 9.9 and negative likelihood ratio 0.8, with very poor performance [AUC 0.58 (95\% CI 0.50-0.67)] and 75.3\% were correctly classified. APRI value of 1 (low threshold) correctly classified $81.6 \%$ of patients and had sensitivity $48.4 \%$, specificity $95 \%$, positive predictive value $79.5 \%$, negative predictive value $82.1 \%$, positive likelihood ratio 9.6 and negative likelihood ratio 0.5 (see Table 2). This value had moderate performance with an AUC at 0.72 (95\% CI 0.630.80 ) and revealed to be the optimal cut-off in defining cirrhosis in our patients. The respective ROC curves of APRI with the AUROC value is shown in Fig. 1.

Performance of FIB-4 scores for the diagnosis of cirrhosis Using optimal cut-off of FIB-4 in previous studies, a value of 3.25 (high threshold) correctly classified $77.1 \%$ of patients with sensitivity $23.4 \%$, specificity $98.7 \%$, positive predictive value $88.2 \%$, negative predictive value

Table 2 Performances of APRI and FIB-4 in predicting cirrhosis using transient elastography as a gold standard

\begin{tabular}{lll}
\hline & APRI 1 & FIB-4 1.66 \\
\hline Se $(95 \% \mathrm{Cl})$ & $48.4 \%(35.7-61.2)$ & $53.1 \%(40.2-65.7)$ \\
Sp $(95 \% \mathrm{Cl})$ & $95.0 \%(90.3-97.8)$ & $92.5 \%(87.1-96.0)$ \\
PPV $(95 \% \mathrm{Cl})$ & $79.5 \%(65.3-88.8)$ & $73.9 \%(61.0-83.6)$ \\
NPV $(95 \% \mathrm{Cl})$ & $82.1 \%(78.3-85.3)$ & $83.1 \%(79.0-86.4)$ \\
LR+ $(95 \% \mathrm{Cl})$ & $9.6(4.7-19.8)$ & $7.0(3.9-12.7)$ \\
LR- $(95 \% \mathrm{Cl})$ & $0.5(0.4-0.7)$ & $0.5(0.3-0.6)$ \\
AC $(95 \% \mathrm{Cl})$ & $81.6 \%(75.9-86.5)$ & $81.1 \%(75.4-86.0)$ \\
AUC $(95 \% \mathrm{Cl})$ & $0.72(0.63-0.80)$ & $0.73(0.65-0.81)$ \\
\hline
\end{tabular}

Se: sensitivity; Sp: specificity; PPV: positive predictive value; NPV: negative predictive value; LR-: negative likelihood ratio; LR+: positive likelihood ratio; Ac: accuracy; K: kappa; AUROC: area under the receiving operator curve
76.2\%, positive likelihood ratio 18.6 and negative likelihood ratio 0.8 in diagnosing liver cirrhosis. The performance was poor with an AUC at 0.61 (95\% CI 0.52-0.70). Lower FIB-4 cut-off 1.94 correctly classified $79.3 \%$ of patients with sensitivity $37.5 \%$, specificity $96.2 \%$, positive predictive value $80 \%$, negative predictive value $79.2 \%$, positive likelihood ratio 9.9, and negative likelihood ratio 0.6. The performance of FIB-4 cut-off 1.94 was also poor with an AUC at 0.67 (95\% CI 0.56-0.76). The optimal cut-off FIB4 score for diagnosing cirrhosis in our patients was 1.66. As summarized in Table 2, at this cut-off, FIB-4 has sensitivity $53.1 \%$, specificity $92.5 \%$, positive predictive value $73.9 \%$, negative predictive value $83.1 \%$, positive likelihood ratio 7.0, and negative likelihood ratio 0.5 . This FIB-4 new cut-off had better performance with AUC at 0.73 (95\% CI $0.65-0.81$ ) and $81.1 \%$ of patients were correctly classified. The ROC curves of FIB-4 with the AUROC value is shown in Fig. 1.

\section{DISSCUSSION}

In our study, low threshold of APRI (1) demonstrate moderate performance [AUC 0.72 (95\% CI 0.63-0.80)] in diagnosing cirrhosis. This cut-off was lower than cutoff use in Mazzola, et al. study (cut-off 2) with moderate performance (AUC 0,77) but almost similar with Merli, et al. study (cut off 0.97) with better performance [AUC 0.84 (95\% 0.81-0.88)]. One third of population in the Merli, et al. study had cirrhosis as in our study, while prevalence of cirrhosis in Mazzola, et al. study was $49.6 \%[13,14]$. Low APRI threshold appeared to be better in diagnosis rather than detecting cirrhosis since the positive predictive value was good (79.5\%), specificity was excellent (95\%), and positive likelihood ratio was good (9.6). Using this threshold, $81.6 \%$ of the patients were correctly classified.

The optimal cut-off of FIB-4 in our population 1.66 was much lower than other studies. Merli, et al. used FIB-4 cut-off 2.02 and Mazzola, et al. used 5.88 for confirming cirrhosis $[13,14]$. Our new cut-off shown a moderate performance with AUC 0.73 (95\% CI 0.65-0.81). This new FIB-4 -cut-off was also better in diagnosis cirrhosis with the positive predictive value, specificity and positive likelihood ratio were good (73.9, 92.5\%, 7.0, respectively). Using this new cut-off, we accurately classified cirrhosis and non-cirrhosis $81.1 \%$ of our population.

To achieve elimination goal, there is an urgent need to limit the cost for diagnostic procedure before starting $\mathrm{HCV}$ therapy, especially in resource-limited setting. In Indonesia only few TE machines are available, mainly located in big cities, and the cost is still high. Based on previous report from Indonesia, an average cost before starting HCV therapy was USD 191, including USD 74 for TE examination [2]. This high cost could delay treatment initiation for many patients though government 


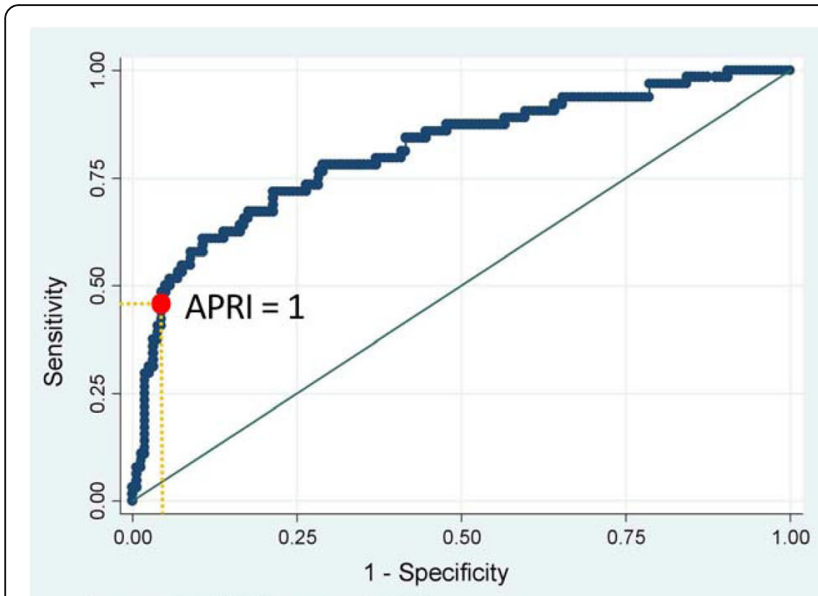

Area under ROC curve $=0.8112$

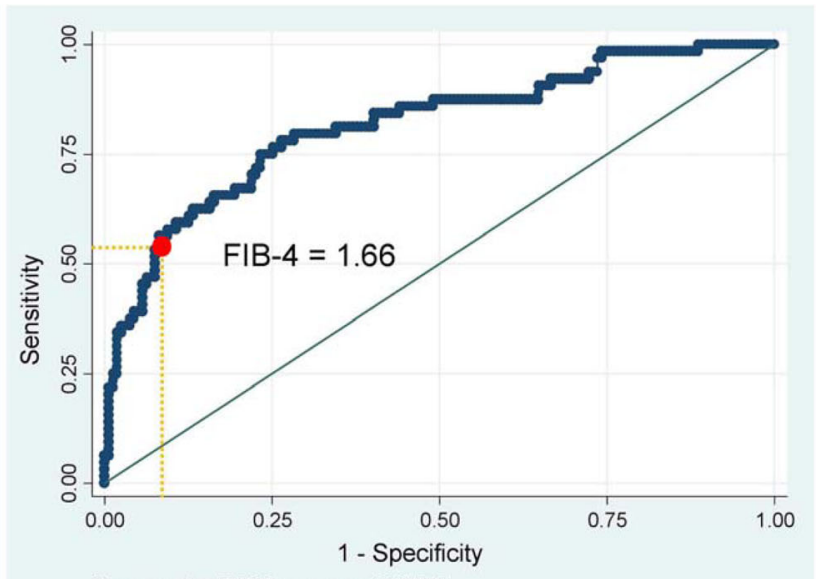

Area under ROC curve $=0.8151$

Fig. 1 ROCs of transient elastography - APRI (left) and transient elastography - FIB-4 (right) of cirrhosis. ROC: receiving operating characteristic curve

provide the drugs. As the majority of $\mathrm{HCV}$ infection in Indonesia were driven by unsafe opiate injection around year 2000, we could predict that many untreated HCVHIV co-infected patients have already in their cirrhosis stage $[15,16]$. HIV co-infection could accelerate the developing of serious liver disease in one third of persons 20 years or less after infection [17]. About 15\% of HCV advanced liver disease patients who did not initiate DAA treatment in the Chronic Hepatitis Cohort Study (CHeCS) had died [18].

Simple and accurate liver fibrosis assessment other than TE examination that can be done in remote area is essential [19, 20]. Since the Indonesian government planned to expand free DAA program for $\mathrm{HCV}$, the result our study could be implemented in many places, as WHO also recommends to use APRI and FIB-4 in resource-constraint countries $[2,14]$.

This study has several limitations. First, the number of end-stage liver diseases were small. This may be caused by the recruited patients came from outpatient clinic where we rarely found end-stage liver disease stage. Quang $\mathrm{Li}$, et al. demonstrated similar limitation among chronic infection of hepatitis B virus (HBV), the sample proportion were inappropriate, merely $7.2 \%$ cirrhotic-patient were cirrhosis [20]. Second, we did not use liver biopsy as gold standard in defining liver cirrhosis. However, our study actually shown a real-world data of TE comparison with these indirect biochemical markers, especially in Asia. Although TE is good validated in HCV infected patients, TE result could be interfered by confounding factors such as hepatic inflammation, hepatic congestion, extrahepatic cholestasis, and liver steatosis. Moreover, we did not investigate performance of biochemical markers against liver biopsy [19]. Third, we need to carefully interpret this result in female patients, since men represent majority of the study population (91.5\%), as observed in other studies [14].

Fourth, the use of cut off of APRI and FIB-4 from this study could only be applied among compensated cirrhosis patients. Perez-Latorre, et al. mentioned decompensated cirrhosis patients have several activation of neurohormonal mechanism, leading to organ inflammatory damage, and decrease accuracy of TE [21]. Therefore, using $\mathrm{TE}$ in liver decompensated must be done carefully.

Fifth, we assessed the diagnostic performances of biochemical markers of fibrosis using TE as a gold standard although we could not validate its performances against liver biopsy in our study population. We recommend further cohort study and larger sample to get new optimal and valid cut-off of APRI and FIB-4.

\section{Conclusion}

APRI and FIB-4 have moderate performance with high specificity in diagnosing cirrhosis. These biochemical markers could be used as diagnosis while TE is not available. In situation where TE is not available, our data support the use of these low-cost biochemical markers as diagnostic tools before DAA treatment.

\section{Abbreviations}

Ac: Accuracy; APRI: Aspartate aminotransferase to platelet ratio index; ART: Antiretroviral therapy; AUC: Area under curve; DAA: Direct acting antiviral; FIB-4: Fibrosis-4; GUCl: Göteborg university cirrhosis index; HBV: Hepatitis B virus; HCV: Hepatitis C virus; HIV: Human immunodeficiency virus; IVDU: Intravenous drug user; LR-: Negative likelihood ratio; LR + : Positive likelihood ratio.; LSM: Liver stiffness measurement; NPV: Negative predictive value; PPV: Positive predictive value; Se: Sensitivity; Sp: Specificity; SVR: Sustained virological response; TE: Transient elastography; WHO: World health organization 


\section{Acknowledgements}

The authors would like to thank Indonesian government, Cipto Mangunkusumo hospital, research team and all patients who were participated in this study.

\section{Authors' contributions}

Conceptualization, EY, BM and AW; Data Curation, EY, BW and AW; Formal Analysis, AJH, BW and AW; Funding Acquisition, EY; Investigation, FA, AW, ASS, JK and VN; Methodology, EY; Project Administration, EY and VN; Resources, EY; Software, AJH; Supervision, EY, AW, ASS and JK; Validation, EY and AW; Visualization EY, AJH, BW and AW. Writing-Original Draft Preparation, EY, BW and AW; Writing-Review \& Editing, EY, BW, AW, ASS and JK. All authors have reviewed and approved the submission of manuscript.

\section{Funding}

The authors declare no fund received for this study.

\section{Availability of data and materials}

The data could be obtained by request to author's email (evy. yunihastuti@gmail.com).

\section{Ethics approval and consent to participate}

Ethical clearance was obtained from Ethical Committee of Faculty Medicine of University of Indonesia and local permission was acquired from Cipto Mangunkusumo hospital authority.

\section{Consent for publication}

Not applicable.

\section{Competing interests}

The authors declare no competing interests for this study.

Received: 26 December 2019 Accepted: 4 May 2020

Published online: 25 May 2020

\section{References}

1. World Health Organization. Hepatitis C [Internet]. Available from: https://www.who.int/en/news-room/fact-sheets/detail/hepatitis-c. Accessed 2019 Aug 15.

2. Trickey A, Hiebert L, Perfect C, Thomas C, LuC J, Kaim E, et al. Hepatitis C virus elimination in Indonesia: epidemiological, cost and cost-effectiveness modelling to advance advocacy and strategic planning. Liver Int. 2019;40(2): 286-97.

3. World Health Organization. Progress report on access to hepatitis $C$ treatment: focus on overcoming barriers in low- and middle-income countries, march 2018. Geneva: World Health Organization; 2018.

4. Lazarus JV, Peric JM, Picchio C, Dillon JF, Robinson M, Cernosa J, et al. We know DAAs work, so now what? Simplifying models of care to enhance the hepatitis C cascade. J Int Med. 2019;286:503-25.

5. Clinton health access intiative. Press Release: $\mathrm{CHAl}$ announces partnership with governments in six low- and middle-income countries to initiate and expand hepatitis C treatment programs. [Internet]. Available from: https:// clintonhealthaccess.org/press-273 release-quick-start/. Accessed 2019 Nov 6.

6. Wyles DL, Ruane PJ, Sulkowski MS, Dieterich D, Luetkemeyer A, Morgan TR, et al. Daclatasvir plus Sofosbuvir for HCV in patients coinfected with HIV-1. N Engl J Med. 2015:1-12.

7. Sandrin L, Fourquet B, Hasquenoph J-M, Yon S, Fournier C, Mal F, et al. Transient elastography: a new noninvasive method for assessment of hepatic fibrosis. Ultrasound Med Biol. 2003;29(12):1705-13.

8. Njei B, McCarty TR, Luk J, Ewelukwa O, Ditah I, Lim JK. Use of transient elastography in patients with HIV-HCV coinfection: a systematic review and meta-analysis. J Gastroenterol Hepatol. 2016;31(10):1684-93.

9. World Health Organization. Guidelines for the screening, care and treatment of persons with chronic hepatitis c infection. Geneva: World Health Organization; 2016.

10. Lucidarme D, Juliette F, Le Bail B, Costera L, Villars S, Forzy G, et al. The ratio interquartile range/median value of liver stiffness measurement is a key factor of accuracy of transient elastography (FibroScan (R)) for the diagnosis of liver fibrosis. Hepatology. 2007 Oct 1;46:318A.

11. Truong TN, Laureillard D, Lacombe K, Thi HD, Hanh PPT, Xuan LTT, et al. High proportion of HIV-HCV coinfected patients with advanced liver fibrosis requiring hepatitis $C$ treatment in Haiphong, northern Vietnam (ANRS 12262). PLoS One. 2016;11(5):1-13.

12. Schmid P, Bregenzer A, Huber M, Rauch A, Jochum W, Müllhaupt B, et al. Progression of liver fibrosis in HIV/HCV co-infection: a comparison between non-invasive assessment methods and liver biopsy. PLoS One. 2015;10(9):1-18.

13. Mazzola G, Adamoli L, Calvaruso V, Macaluso FS, Colletti P, Mazzola S, et al. Suboptimal performance of APRI and FIB-4 in ruling out significant fibrosis and confirming cirrhosis in HIV/HCV co-infected and HCV mono-infected patients. Infection. 2019;47(3):409-15.

14. Merli M, Galli L, Castagna A, Salpietro S, Gianotti N, Messina E, et al. Diagnostic accuracy of APRI, FIB-4 and Forns for the detection of liver cirrhosis in HIV/HCV-coinfected patients. New Microbiol. 2016;39(2):110-3.

15. Ghys PD, Bazant W, Monteiro MG, Calvani S, Lazzari S. The epidemics of injecting drug use and HIV in Asia. AIDS. 2001;15(suppl 5):S91-9.

16. World Health Organization. Country profile: Indonesia. In: atlas of substance use disorders. Geneva: World Health Organization; 2010. p. 1-5.

17. Lauer GM, Walker BD. Hepatitis C virus infection. N Engl J Med. 2001; 345(1):41-52.

18. Spradling PR, Xing J, Rupp LB, Moorman AC, Gordon SC, Lu M, et al. Low uptake of direct-acting antiviral therapy among hepatitis $C$ patients with advanced liver disease and access to care, 2014-2017. J Clin Gastroenterol. 2020;Volume Pub (Issue):doi: https://doi.org/10.1097/MCG. 0000000000001344

19. Yen YH, Kuo FY, Kee KM, Chang KC, Tsai MC, Hu TH, et al. APRI and FIB-4 in the evaluation of liver fibrosis in chronic hepatitis $C$ patients stratified by AST level. PLoS One. 2018;13(6):1-16.

20. Li Q, Ren X, Lu C, Li W, Huang Y, Chen L. Evaluation of APRI and FIB-4 for noninvasive assessment of significant fibrosis and cirrhosis in $\mathrm{HBeAg}$ negative $\mathrm{CHB}$ patients with $\mathrm{ALT} \leq 2 \mathrm{ULN}$ a retrospective cohort study. Medicine. 2017;96(12):1-7.

21. Pérez-latorre $L$, Sánchez-conde $M$, Rincón D, Miralles $P$, Aldámiz-echevarría $T$, Carrero A, et al. Prediction of liver complications in patients with hepatitis $C$ virus - related cirrhosis with and without HIV coinfection: comparison of hepatic venous pressure gradient and transient elastography. Clin Infect Dlis. 2014;58:713-8.

\section{Publisher's Note}

Springer Nature remains neutral with regard to jurisdictional claims in published maps and institutional affiliations.

Ready to submit your research? Choose BMC and benefit from:

- fast, convenient online submission

- thorough peer review by experienced researchers in your field

- rapid publication on acceptance

- support for research data, including large and complex data types

- gold Open Access which fosters wider collaboration and increased citations

- maximum visibility for your research: over $100 \mathrm{M}$ website views per year

At BMC, research is always in progress.

Learn more biomedcentral.com/submissions 